\title{
ARTICLE
}

Clinical Study

\section{Long-term follow-up of overall survival for cabozantinib versus everolimus in advanced renal cell carcinoma}

\author{
Robert J. Motzer ${ }^{1}$, Bernard Escudier ${ }^{2}$, Thomas Powles ${ }^{3}$, Christian Scheffold ${ }^{4}$ and Toni K. Choueiri (iD ${ }^{5}$
}

BACKGROUND: In the phase 3 METEOR trial (NCT01865747), cabozantinib significantly improved progression-free survival, overall survival, and objective response rate compared with everolimus in patients with advanced renal cell carcinoma (RCC) after prior antiangiogenic therapy. A statistically significant improvement in overall survival was observed at a second interim analysis with 320 recorded deaths.

METHODS: 658 patients with advanced RCC who had received at least one prior VEGFR tyrosine kinase inhibitor were randomised 1:1 to cabozantinib ( $60 \mathrm{mg}$ daily) or everolimus ( $10 \mathrm{mg}$ daily). Survival follow-up continued to reach the 408 deaths that were prespecified for the final analysis.

RESULTS: With 430 deaths (198 for cabozantinib and 232 for everolimus), median overall survival was 21.4 months with cabozantinib and 17.1 months with everolimus (HR $0.70,95 \% \mathrm{Cl} 0.58-0.85 ; P=0.0002$ ). Safety profiles of cabozantinib and everolimus were consistent with those reported previously.

CONCLUSIONS: Cabozantinib significantly improved overall survival compared with everolimus in previously treated patients with advanced RCC with consistent results after long-term follow-up.

British Journal of Cancer (2018) 118:1176-1178; https://doi.org/10.1038/s41416-018-0061-6

\section{INTRODUCTION}

Cabozantinib is an oral inhibitor of tyrosine kinases including MET, vascular endothelial growth factor (VEGF) receptors, and AXL. ${ }^{1}$ Cabozantinib is approved for the treatment of patients with advanced renal cell carcinoma (RCC) after prior antiangiogenic therapy based on results of the phase 3 METEOR trial showing significant improvements in progression-free survival, overall survival, and objective response rate compared with everolimus. ${ }^{2,3}$ At the second interim analysis with 320 (78\%) of the 408 planned events and a minimum follow-up of 13 months, the null hypothesis of no difference in overall survival was rejected. Differences in overall survival were statistically significant with a median of 21.4 months with cabozantinib versus 16.5 months with everolimus (hazard ratio [HR] $0.66,95 \% \mathrm{Cl}$ $0.53-0.83 ; P=0.0003$ ). Subgroup analyses of overall survival were consistent with results for the overall population. We report results for overall survival and updated safety after follow-up was continued to reach the number of deaths pre-specified for the final analysis.

\section{MATERIALS AND METHODS}

METEOR is a randomised, open-label, phase 3 trial with patients enrolled at 173 centres in 26 countries. ${ }^{2,3}$ Eligible patients were 18 years of age or older with advanced or metastatic clear-cell RCC and measurable disease per Response Evaluation Criteria in Solid
Tumors (RECIST version 1.1). ${ }^{4}$ Patients must have received at least one prior VEGFR tyrosine kinase inhibitor (TKI) and must have progressed within 6 months of their most recent VEGFR TKI and within 6 months of randomisation. A Karnofsky performance status of at least 70 was required.

Patients were randomised 1:1 to cabozantinib (60 mg once daily) or everolimus ( $10 \mathrm{mg}$ once daily). Randomisation was stratified by Memorial Sloan Kettering Cancer Center (MSKCC) risk group and number of prior VEGFR TKIs (1 versus 2 or more). Dose reduction levels to manage adverse events were 40 and 20 $\mathrm{mg}$ for cabozantinib and 5 and $2.5 \mathrm{mg}$ for everolimus. To maximise the ability to evaluate the effect of study treatment on overall survival, treatment crossover was not allowed in the study. The study was conducted according to the Good Clinical Practice guidelines and the Declaration of Helsinki. The protocol was approved by the institutional review board or ethics committee at each centre, and written informed consent was obtained for all patients.

The primary endpoint of progression-free survival and secondary endpoints of overall survival and objective response rate and safety have been reported previously. ${ }^{2,3}$ After determination of the primary endpoint, tumour scans were no longer centrally collected nor analysed in aggregate. Safety, including adverse events, was evaluated every 2 weeks for the first 8 weeks and every 4 weeks thereafter until treatment discontinuation. Adverse events were graded according to Common Terminology Criteria

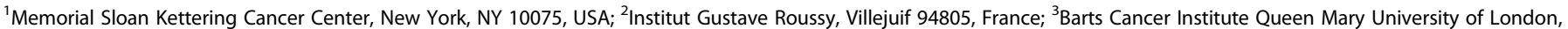
London EC1M 6BQ, UK; ${ }^{4}$ Exelixis, Inc, South San Francisco, CA 94080, USA and ${ }^{5}$ Dana-Farber Cancer Institute, Boston, MA 02215, USA

Correspondence: Robert J. Motzer (motzerr@MSKCC.ORG)

Received: 1 December 2017 Revised: 15 February 2018 Accepted: 28 February 2018

Published online: 26 March 2018 
or Adverse Events version 4.0. Patients were followed for overall survival every 8 weeks. Information on subsequent anticancer therapy was also collected.

Overall survival was analysed in all 658 randomised patients. For overall survival, 408 deaths were required to detect a HR of 0.75 (80\% power, two-sided $a=0.04$ ) with a hypothesised improvement in median survival from 15 months to 20 months and one planned interim analysis to be conducted at the time of the primary endpoint analysis of progression-free survival. The first planned interim analysis did not meet the criteria for significance defined by the Lan-DeMets O'Brien-Fleming alpha spending function. However, the criteria for significance for rejection of the null hypothesis was met at a second interim analysis conducted with a prospectively defined cutoff date of 13 December 2015 and a minimum follow-up of 13 months $(P=$ 0.0003 ; critical $P$-value $\leq 0.0163) .{ }^{2,3}$ Follow-up was continued to reach the 408 deaths pre-specified for the final analysis to collect long-term survival data.

Hypothesis testing of overall survival was performed using the stratified log-rank test with the randomisation stratification factors. Subgroup analyses of overall survival used all randomised patients and were pre-specified; HRs for subgroup analyses are unstratified and confidence intervals are considered descriptive.

\section{RESULTS}

A total of 658 patients were randomised from August 2013 to November 2014 (330 to cabozantinib and 328 to everolimus). Baseline characteristics were balanced between treatment arms. ${ }^{2}$,

${ }^{3}$ The majority of patients were male (75\%) with a median age of 62 years. Forty-six percent of patients were in the favourable risk

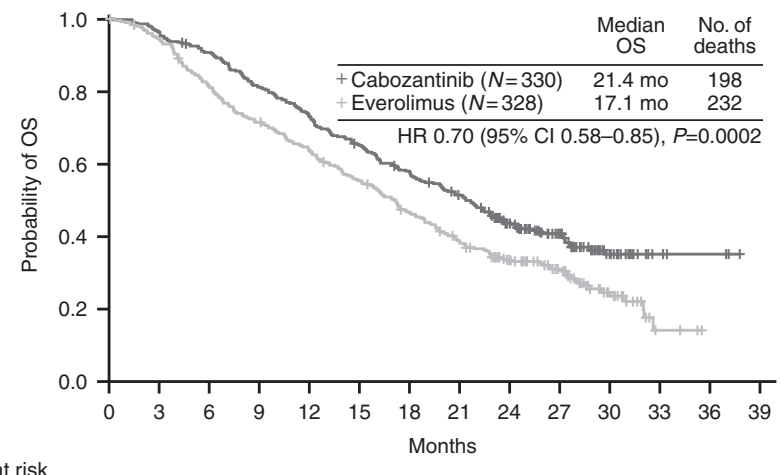

No. at risk

Cabozantinib $\begin{array}{lllllllllllllll}330 & 318 & 297 & 266 & 240 & 213 & 188 & 163 & 114 & 76 & 30 & 6 & 3 & 0\end{array}$ $\begin{array}{lllllllllllllll}\text { Everolimus } & 328 & 308 & 264 & 231 & 205 & 177 & 148 & 121 & 82 & 54 & 23 & 3 & 0 & 0\end{array}$

Fig. 1 Overall survival through 2 October 2016. HR hazard ratio, OS overall survival category, $42 \%$ in the intermediate risk category, and $13 \%$ in the poor risk category as defined by MSKCC prognostic criteria for RCC.

As of the 2 October 2016 data cutoff for overall survival, 11\% (36/330) of patients in the cabozantinib group and $2.4 \%(8 / 328)$ of patients in the everolimus group remained on study treatment. The minimum follow-up was 22 months, median follow-up was 28 months (IQR 25, 30), and 430 deaths were recorded (198 for cabozantinib and 232 for everolimus). Analysis of overall survival showed a significant improvement for cabozantinib compared with everolimus: median overall survival was 21.4 months with cabozantinib and 17.1 months with everolimus (HR $0.70,95 \% \mathrm{Cl}$ $0.58-0.85 ; P=0.0002$ ) (Fig. 1). Overall survival landmark estimates for cabozantinib and everolimus were $44 \%$ versus $34 \%$ at 24 months and $35 \%$ versus $25 \%$ at 30 months. Overall survival analyses of subgroups defined by stratification factors were consistent with results for the overall population (Fig. 2). Use of systemic subsequent anticancer therapy was $57 \%$ versus $63 \%$ for cabozantinib versus everolimus and included VEGFR TKIs ( $28 \%$ for cabozantinib versus $50 \%$ for everolimus), everolimus (33\% versus $5 \%)$, PD- 1 checkpoint inhibitors (14\% versus $16 \%$, with $13 \%$ versus $15 \%$ receiving nivolumab), and cabozantinib (1\% versus $4 \%$ ).

The safety population consisted of 331 cabozantinib-treated patients and 322 everolimus-treated patients. As of the 2 October 2016 data cutoff, the median duration of exposure was 8.4 months (range, 0.3-37.0) for cabozantinib-treated patients versus 4.4 months (range, 0.2-32.2) for everolimus-treated patients. Dose reductions due to adverse events were implemented in $64 \%$ of cabozantinib-treated patients and $25 \%$ of everolimus-treated patients. The median average daily dose was $42.8 \mathrm{mg}$ for cabozantinib and $9.1 \mathrm{mg}$ for everolimus. Discontinuation due to adverse events occurred for 43 (13\%) cabozantinib-treated patients and 35 (11\%) everolimus-treated patients.

All patients in the cabozantinib group and all but one patient in the everolimus group experienced an adverse event of any grade irrespective of causality; grade 3 or 4 adverse events were experienced by $71 \%$ of cabozantinib-treated patients and $61 \%$ of everolimus-treated patients (Table 1). Grade 5 events occurred for 31 (9\%) cabozantinib-treated patients and 26 (8\%) everolimustreated patients. One grade 5 adverse event was treatmentrelated in the cabozantinib group (death), and two were treatment-related in the everolimus group (aspergillus infection and pneumonia aspiration).

\section{DISCUSSION}

This study reports long-term survival data with a minimum followup of 22 months from the pivotal phase 3 METEOR trial of cabozantinib versus everolimus in patients with previously treated advanced RCC. Results show a significant improvement in overall

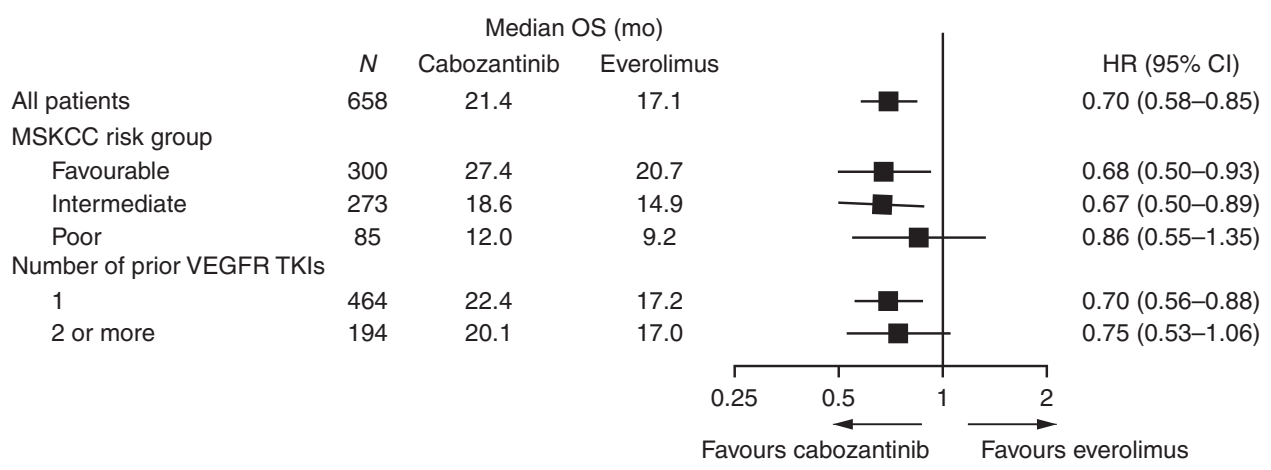

Fig. 2 Forest plots of overall survival through 2 October 2016. All randomised patients were included in the analyses. All HRs are unstratified except for the analysis in all patients. HR hazard ratio, MSKCC Memorial Sloan Kettering Cancer Center, OS overall survival, TKI tyrosine kinase inhibitor, VEGFR vascular endothelial growth factor receptor 
Table 1. All causality grade 3 or 4 adverse events

\begin{tabular}{lll}
\hline & $\begin{array}{l}\text { Cabozantinib } \\
(N=331)\end{array}$ & $\begin{array}{l}\text { Everolimus } \\
(N=322)\end{array}$ \\
\hline Any adverse event, $n(\%)$ & $236(71)$ & $196(61)$ \\
Hypertension & $51(15)$ & $12(4)$ \\
Diarrhoea & $44(13)$ & $8(2)$ \\
Fatigue & $36(11)$ & $24(7)$ \\
PPE & $28(8)$ & $3(1)$ \\
Anaemia & $22(7)$ & $55(17)$ \\
Hypokalaemia & $16(5)$ & $6(2)$ \\
Hypomagnesaemia & $16(5)$ & 0 \\
Hyponatraemia & $16(5)$ & $8(2)$ \\
Nausea & $16(5)$ & $1(<1)$ \\
Asthaenia & $15(5)$ & $8(2)$ \\
Hyperglycaemia & $3(1)$ & $16(5)$ \\
\hline Adverse
\end{tabular}

Adverse events that occurred at $\geq 5 \%$ in either treatment arm are summarised. Patients are counted once at the highest grade for each preferred term. The severity of adverse events was graded according to the National Cancer Institute Common Terminology Criteria for Adverse Events (version 4.0). PPE palmar-plantar erythrodysesthesia

survival for cabozantinib compared with everolimus that was consistent with the earlier analysis in which the null hypothesis was rejected. With 9 additional months of follow-up, median survival was 21.4 months with cabozantinib and 17.1 months with everolimus (HR 0.70) compared with 21.4 months with cabozantinib and 16.5 months with everolimus (HR 0.66) at the earlier analysis. ${ }^{2}$ Subgroup analyses of overall survival based on the stratification factors were consistent with results for the overall population showing a survival benefit with cabozantinib compared with everolimus, similar to previous results. ${ }^{2}$

The PD-1 checkpoint inhibitor nivolumab has also shown a benefit in overall survival in a similar patient population when compared with everolimus. ${ }^{5}$ In the nivolumab study, long-term survival data at 3 years showed a HR of $0.74(95.45 \% \mathrm{Cl} 0.63-0.88){ }^{6}$

A possible limitation of analysing survival outcomes with longer follow-up is that increased use of subsequent systemic therapy may confound the results. The percent of patients who received subsequent systemic therapy was similar in the two treatment groups; however, the type of subsequent therapy received differed. Patients in the cabozantinib group more frequently received subsequent everolimus, while patients in the everolimus group more frequently received subsequent therapy with VEGFR TKIs. Importantly, subsequent therapy with the PD-1 checkpoint inhibitor nivolumab, which has demonstrated prolongation of overall survival in this patient population, ${ }^{5}$ was similar in both treatment groups.

The safety profiles of cabozantinib and everolimus were consistent with those reported previously, ${ }^{2}$ with similar reported incidences of adverse events, dose reductions, and discontinuations due to adverse events. The similar results reflect both the reduced number of patients on study treatment and continued tolerability for those remaining on treatment.

Cabozantinib significantly improved overall survival compared with everolimus in previously treated patients with advanced RCC with a consistent benefit after long-term follow-up.

\section{ACKNOWLEDGEMENTS}

We thank the patients, their families, the investigators and site staff, and the study teams participating in this trial. The study was funded by Exelixis, Inc. Patients treated at Memorial Sloan Kettering Cancer Center were supported in part by Memorial Sloan Kettering Cancer Center Support Grant/Core Grant (P30 CA008748). Medical writing support was provided by Julie C. Lougheed, PhD (Exelixis, Inc.), with editorial assistance by Fishawack Communications (Conshohocken, PA, USA), which was funded by Exelixis.

\section{AUTHOR CONTRIBUTIONS}

Study conception and design: R.J.M., T.K.C., B.E., T.P., and C.S. Patient recruitment: R.J. M., T.K.C., B.E., and T.P. Data collection, interpretation, and writing of the manuscript: all authors.This study is sponsored by Exelixis.

\section{ADDITIONAL INFORMATION}

Competing interests: R.M. has received personal fees for an advisory/consulting role from Pfizer, Novartis, Eisai, Exelixis, and research funding from Exelixis and from Bristol-Myers Squibb, Pfizer, Genentech/Roche, Eisai, Novartis, outside the submitted work. B.E. has received personal fees for an advisory/consulting role from Novartis, Bristol-Myers Squibb, Roche, Exelixis, Ipsen, Acceleron Pharma, Bayer, and research funding from Novartis and Bristol-Myers Squibb, outside the submitted work. T.P. has received personal fees for an advisory/consulting role from AstraZeneca, BristolMyers Squibb, Genentech/Roche, Merck, Exelixis and research funding from AstraZeneca/Medlmmune, Roche/Genentech, outside the submitted work. C.S. is an employee of Exelixis. T.C. has received personal fees for an advisory/consulting role from Pfizer, GlaxoSmithKline, Novartis, Merck, Bayer, Eisai, Roche, Prometheus Labs Inc, Foundation Medicine Inc., Bristol-Myers Squibb, and institutional research funding from Exelixis and from Pfizer, GlaxoSmithKline, Novartis, Bristol-Myers Squibb, Merck, Roche, AstraZeneca, Tracon, and Peloton, outside the submitted work.

Ethics approval and consent to participate: The study was conducted according to the Good Clinical Practice guidelines and the Declaration of Helsinki. The protocol was approved by the institutional review board or ethics committee at each centre, and written informed consent was obtained for all patients.

Funding: This study is sponsored by Exelixis.

\section{REFERENCES}

1. Yakes, F. M. et al. Cabozantinib (XL184), a novel MET and VEGFR2 inhibitor, simultaneously suppresses metastasis, angiogenesis, and tumor growth. Mol. Cancer Ther. 10, 2298-2308 (2011).

2. Choueiri, T. K. et al. Cabozantinib versus everolimus in advanced renal cell carcinoma (METEOR): final results from a randomised, open-label, phase 3 trial. Lancet Oncol. 17, 917-927 (2016).

3. Choueiri, T. K. et al. Cabozantinib versus everolimus in advanced renal-cell carcinoma. N. Engl. J. Med. 373, 1814-1823 (2015).

4. Eisenhauer, E. A. et al. New response evaluation criteria in solid tumours: revised RECIST guideline (version 1.1). Eur. J. Cancer 45, 228-247 (2009).

5. Motzer, R. J. et al. Nivolumab versus everolimus in advanced renal-cell carcinoma. N. Engl. J. Med. 373, 1803-1813 (2015).

6. Bristol-Meyers Squibb Press Release: Opdivo (nivolumab) demonstrates superior three-year survival benefit for patients with previously treated advanced renal cell carcinoma (RCC). https://news.bms.com/press-release/corporatefinancial-news/ opdivo-nivolumab-demonstrates-superior-three-year-survival-ben. Accessed 7 Nov 2017 (posted 6 Nov 2017).

Open Access This article is licensed under a Creative Commons Attribution 4.0 International License, which permits use, sharing, adaptation, distribution and reproduction in any medium or format, as long as you give appropriate credit to the original author(s) and the source, provide a link to the Creative Commons license, and indicate if changes were made. The images or other third party material in this article are included in the article's Creative Commons license, unless indicated otherwise in a credit line to the material. If material is not included in the article's Creative Commons license and your intended use is not permitted by statutory regulation or exceeds the permitted use, you will need to obtain permission directly from the copyright holder. To view a copy of this license, visit http://creativecommons. org/licenses/by/4.0/.

(c) The Author(s) 2018 